\title{
FORWARD MODELING OF STANDING SLOW MODES IN FLARING CORONAL LOOPS
}

\author{
D. Yuan ${ }^{1,2}$, T. Van Doorsselaere ${ }^{1}$, D. BanerjeE ${ }^{3}$, and P. Antolin ${ }^{4}$ \\ ${ }^{1}$ Centre for mathematical Plasma Astrophysics, Department of Mathematics, KU Leuven, Celestijnenlaan 200B bus 2400, \\ B-3001 Leuven, Belgium; Ding.Yuan@wis.kuleuven.be \\ ${ }^{2}$ Key Laboratory of Solar Activity, National Astronomical Observatories, Chinese Academy of Sciences, Beijing, 100012, China \\ ${ }^{3}$ Indian Institute of Astrophysics, II Block, Koramangala, Bangalore 560 034, India \\ ${ }^{4}$ National Astronomical Observatory of Japan, 2-21-1 Osawa, Mitaka, Tokyo 181-8588, Japan \\ Received 2015 March 3; accepted 2015 May 22; published 2015 July 2
}

\begin{abstract}
Standing slow-mode waves in hot flaring loops are exclusively observed in spectrometers and are used to diagnose the magnetic field strength and temperature of the loop structure. Owing to the lack of spatial information, the longitudinal mode cannot be effectively identified. In this study, we simulate standing slow-mode waves in flaring loops and compare the synthesized line emission properties with Solar Ultraviolet Measurements of Emitted Radiation spectrographic and Solar Dynamics Observatory/Atmospheric Imaging Assembly imaging observations. We find that the emission intensity and line width oscillations are a quarter period out of phase with Doppler shift velocity in both time and spatial domain, which can be used to identify a standing slow-mode wave from spectroscopic observations. However, the longitudinal overtones could only be measured with the assistance of imagers. We find emission intensity asymmetry in the positive and negative modulations; this is because the contribution function pertaining to the atomic emission process responds differently to positive and negative temperature variations. One may detect half periodicity close to the loop apex, where emission intensity modulation is relatively small. The line-of-sight projection affects the observation of Doppler shift significantly. A more accurate estimate of the amplitude of velocity perturbation is obtained by de-projecting the Doppler shift by a factor of $1-2 \theta / \pi$ rather than the traditionally used $\cos \theta$. If a loop is heated to the hotter wing, the intensity modulation could be overwhelmed by background emission, while the Doppler shift velocity could still be detected to a certain extent.
\end{abstract}

Key words: magnetohydrodynamics (MHD) - Sun: atmosphere - Sun: corona - Sun: oscillations - waves

\section{INTRODUCTION}

Magnetohydrodynamic (MHD) waves are believed to play a significant role in the formation and dynamics of the solar atmosphere. They may contribute significantly to coronal heating (see reviews by Klimchuk 2006; Taroyan \& Erdélyi 2009; Parnell \& De Moortel 2012; Arregui 2015) and solar wind acceleration (see, e.g., Ofman 2010; van der Holst et al. 2014). During the past decade, a number of MHD wave modes of coronal loops were detected with modern instruments, e.g., standing and propagating fast kink mode (Aschwanden et al. 1999; Nakariakov et al. 1999; Williams et al. 2002; Van Doorsselaere et al. 2008), fast sausage modes (Asai et al. 2001; Melnikov et al. 2005), and standing and propagating slow mode (De Moortel et al. 2000, 2002a, 2002b; Wang et al. 2003a, 2003b, 2009a, 2009b; Yuan \& Nakariakov 2012; Krishna Prasad et al. 2014).

MHD wave theory in structured plasma forms a solid basis for a wave-based plasma diagnostic technique-MHD coronal seismology (see Nakariakov \& Verwichte 2005; De Moortel \& Nakariakov 2012, for recent reviews). MHD seismology was successfully applied in estimating the coronal magnetic field (Nakariakov \& Ofman 2001), transverse loop structuring (Aschwanden et al. 2003), Alfvén transit times (Arregui et al. 2007), polytropic index (Van Doorsselaere et al. 2011b), thermal conduction coefficient (Van Doorsselaere et al. 2011b), the magnetic topology of sunspots (Jess et al. 2013; Yuan et al. 2014a, 2014b), and the magnetic structure of large-scale streamers (Chen et al. 2010, 2011). It could also be used to determine the coronal density scale height (Andries et al. 2005), to quantify the expansion factor of the coronal loops (Verth et al. 2008), and to probe the characteristic spatial scale of randomly structured plasmas (Yuan et al. 2015).

The slow sausage mode was initially theorized by Edwin \& Roberts (1983). It is a compressive mode characterized by axisymmetric longitudinal displacement of the plasma fluid. Gas pressure is the main restoring force. In a low- $\beta$ plasma, this mode does not cause significant contraction or expansion of the loop cross section, nor a displacement of the loop axis. Standing slow modes are frequently observed as oscillations in the plasma emission intensity and Doppler shift velocity in hot flaring coronal loops ( $>6 \mathrm{MK}$; see review by Wang 2011). They are exclusively detected in the hot emission lines, i.e., Fe XIX and Fe Xxi lines as recorded by the Solar Ultraviolet Measurements of Emitted Radiation (SUMER) spectrograph on board Solar and Heliospheric Observatory (SOHO; Wang et al. 2002, 2003a, 2003b), and S xv and Ca XIX lines observed by the Bragg Crystal Spectrometer on board Yohkoh (Mariska 2006). Only recently, the Solar Dynamics Observatory (SDO)/Atmospheric Imaging Assembly (AIA) imager observed, in the $131 \AA$ bandpass, multiple reflections of a propagating slow wave in a hot coronal loop (Kumar et al. 2013). A slow compressive mode is found to be launched by repetitive magnetic reconnections occurring at one of the footpoints (Kumar et al. 2015).

Quasi-periodic pulsations in solar and stellar flares are thought to be caused by MHD waves (Nakariakov \& Melnikov 2009; Anfinogentov et al. 2013). Oscillations with periods at tens of minutes are ascribed to modulations by slowmode MHD waves (Van Doorsselaere et al. 2011a), while short-period (sub-minute) oscillations are suggested to be modulated by fast-mode waves (Van Doorsselaere et al. 2011a; Kupriyanova et al. 2013). Recently, Kim et al. (2012) detected 
oscillations during an M1.6 flare with the Nobeyama Radioheliograph and the $335 \AA$ extreme-ultraviolet (EUV) channel of SDO/AIA. The intensities in radio and EUV emissions oscillate with a period of about 13.8 minutes and are damped with a decay time of about 25 minutes. Kim et al. (2012) interpreted the detected 10-minute timescale oscillations as standing slow magnetoacoustic waves. SUMER-like oscillations, but with shorter periodicities, are detected in soft X-ray emissions (Ning 2014).

The standing slow-mode wave oscillates with a period of about 10 minutes and with a velocity amplitude of a few tens of kilometers per second (Wang et al. 2003a; Wang 2011). Significant oscillation is normally detected at the loop apex and becomes absent at the footpoints (Wang et al. 2007). This is consistent with a scenario in which an antinode in the density perturbation of the fundamental standing slow mode is located at the loop apex (Wang et al. 2007). This kind of wave is damped within a few oscillation cycles, which are believed to be caused mainly by thermal conduction in hot flaring loops (Ofman \& Wang 2002; Selwa et al. 2005).

The fundamental standing slow mode of a hot loop appears to be triggered by asymmetric heating at one footpoint and is rapidly established within one wave cycle (Wang et al. 2005). Pressure pulses launched close to a footpoint cannot excite a fundamental slow mode fast enough to compensate the strong damping (Selwa et al. 2005). Taroyan et al. (2005) showed analytically that the fundamental slow mode of a $6 \mathrm{MK}$ loop could rapidly be excited by a single impulsive heating with a timescale that matches the loop period. Forward modeling using a simple one-dimensional hydrodynamic model was performed to distinguish the propagating and standing slow waves in both cool and hot loops (Taroyan et al. 2007; Taroyan \& Bradshaw 2008). They also reported that Doppler shift variation is a more reliable observable to detect a slow wave, while intensity modulation would be phase-shifted by heating (Taroyan et al. 2007) or contaminated by the background plasma emission (Taroyan \& Bradshaw 2008).

MHD seismology with the standing slow mode was applied successfully to estimate the magnetic field strength and the time-dependent plasma temperature of a coronal loop (Wang et al. 2007). The application of MHD seismology relies on several nontrivial factors, e.g., the analytical model, mode identification, line-of-sight (LOS) effect, and plasma emission. Forward modeling was attempted to help interpret the observations correctly. Cooper et al. (2003a, 2003b) investigated the LOS effect on imaging observation of the emission intensity variation of fast kink and sausage mode and explained the intensity perturbations of fast wave trains observed by the Solar Eclipse Coronal Imaging System instrument (Williams et al. 2001, 2002). Gruszecki et al. (2012) performed a threedimensional numerical simulation of the fast sausage mode of a plasma cylinder and investigated the geometric effect of simple LOS integration. Antolin \& Van Doorsselaere (2013) and Antolin et al. (2014) developed advanced models and included atomic emission effects, using the CHIANTI atomic database (Dere et al. 1997). They found that the LOS effect and spatial resolution could significantly affect the intensity modulation and spectral characteristics of the fast sausage mode. Reznikova et al. $(2014,2015)$ used the same model and further investigated the gyrosynchrotron emission intensity variation using the fast gyrosynchrotron codes (Fleishman \& Kuznetsov 2010). The radio emission intensity of the fast sausage mode oscillates in phase for all frequencies, while for certain LOS angles, the optically thick and thin radio emissions are antiphase. It opposes previous findings of Mossessian \& Fleishman (2012), which did not consider the inhomogeneity of the emitting source along the LOS. Kuznetsov et al. (2015) used a semi-torus model to forward model the gyrosynchrotron radio emission of both propagating and standing slow modes in a curved magnetic structure.

In this study, we perform forward modeling of standing slow modes of hot flaring coronal loops and predict their spectroscopic and imaging observational signatures. We use the slowwave model in hot coronal loops (>6 MK; see Section 2) and utilize the CHIANTI v7.1 atomic database (Dere et al. 1997; Landi et al. 2013) to synthesize plasma emission in the SUMER Fe XIX line and SDO/AIA $094 \AA$ bandpass (see Section 3 ). Then the results and conclusions are summarized in Sections 4 and 5, respectively.

\section{MODEL}

\subsection{Standing Slow Mode}

In this study, we only consider a standing slow mode in a simple plasma cylinder embedded in a uniform plasma. The magnetic field is parallel to the axis of the plasma cylinder (i.e., $z$-axis), $\boldsymbol{B}_{\boldsymbol{0}}=B_{0} \hat{z}$. The equilibrium magnetic field $B_{0}$, plasma density $\rho_{0}$, and temperature $T_{0}$ are the piecewise functions of the $r$-axis:

$$
B_{0}, \rho_{0}, T_{0}=\left\{\begin{array}{l}
B_{\mathrm{i}}, \rho_{\mathrm{i}}, T_{\mathrm{i}}: r \leqslant a \\
B_{\mathrm{e}}, \rho_{\mathrm{e}}, T_{\mathrm{e}}: r>a,
\end{array}\right.
$$

where $a$ is the radius of the loop. Hereafter, we use subscripts "i" and "e" to differentiate the internal and external equilibrium values of the loop system.

Effects of plasma stratification and loop curvature are ignored. We focus on observational features caused by optically thin plasma emission, LOS integration, and instrument response function. We limit our study to the axisymmetric mode $m=0$ (sausage mode; see Edwin \& Roberts 1983).

The linearized ideal MHD equations (see, e.g., Ruderman \& Erdélyi 2009) give the perturbed quantities on top of the magnetostatic equilibrium:

$$
\begin{gathered}
\rho_{1}=-\nabla \cdot\left(\rho_{0} \boldsymbol{\xi}\right), \\
\rho_{0} \frac{\partial^{2} \boldsymbol{\xi}}{\partial t^{2}}=-\nabla P_{\mathrm{T} 1}+\frac{1}{\mu_{0}}\left[\left(\boldsymbol{B}_{\boldsymbol{0}} \cdot \nabla\right) \boldsymbol{b}_{\boldsymbol{1}}+\left(\boldsymbol{b}_{\boldsymbol{1}} \cdot \nabla\right) \boldsymbol{B}_{\boldsymbol{0}}\right] \\
\boldsymbol{b}_{\boldsymbol{1}}=\nabla \times\left(\boldsymbol{\xi} \times \boldsymbol{B}_{\mathbf{0}}\right), \\
p_{1}-C_{\mathrm{s}}^{2} \rho_{1}=\boldsymbol{\xi} \cdot\left(C_{\mathrm{s}}^{2} \nabla \rho_{0}-\nabla p_{0}\right),
\end{gathered}
$$

where $\boldsymbol{\xi}$ is the Lagrangian displacement vector; $p_{0}$ is the equilibrium plasma pressure; $\rho_{1}, p_{1}$, and $\boldsymbol{b}_{\boldsymbol{1}}$ are the perturbed plasma density, pressure, and magnetic field, respectively; $P_{\mathrm{T} 1}=p_{1}+\boldsymbol{b}_{\boldsymbol{1}} \cdot \boldsymbol{B}_{\boldsymbol{0}} / \mu_{0}$ is the perturbed total pressure; and $\mu_{0}$ is the magnetic permeability in free space. We define the key characteristic speeds to describe the loop system: $C_{\mathrm{s}}=\sqrt{\gamma p_{0} / \rho_{0}}$, $C_{\mathrm{A}}=B_{0} / \sqrt{\mu_{0} \rho_{0}}$, and $C_{\mathrm{T}}=C_{\mathrm{A}} C_{\mathrm{s}} / \sqrt{C_{\mathrm{A}}^{2}+C_{\mathrm{s}}^{2}}$ are the acoustic, Alfvén, and tube speeds, respectively (Edwin \& Roberts 1983); $\omega_{\mathrm{s}}=C_{\mathrm{s}} k, \omega_{\mathrm{A}}=C_{\mathrm{A}} k$, and $\omega_{\mathrm{T}}=C_{\mathrm{T}} k$ are the corresponding acoustic, Alfvén, and tube frequencies, 


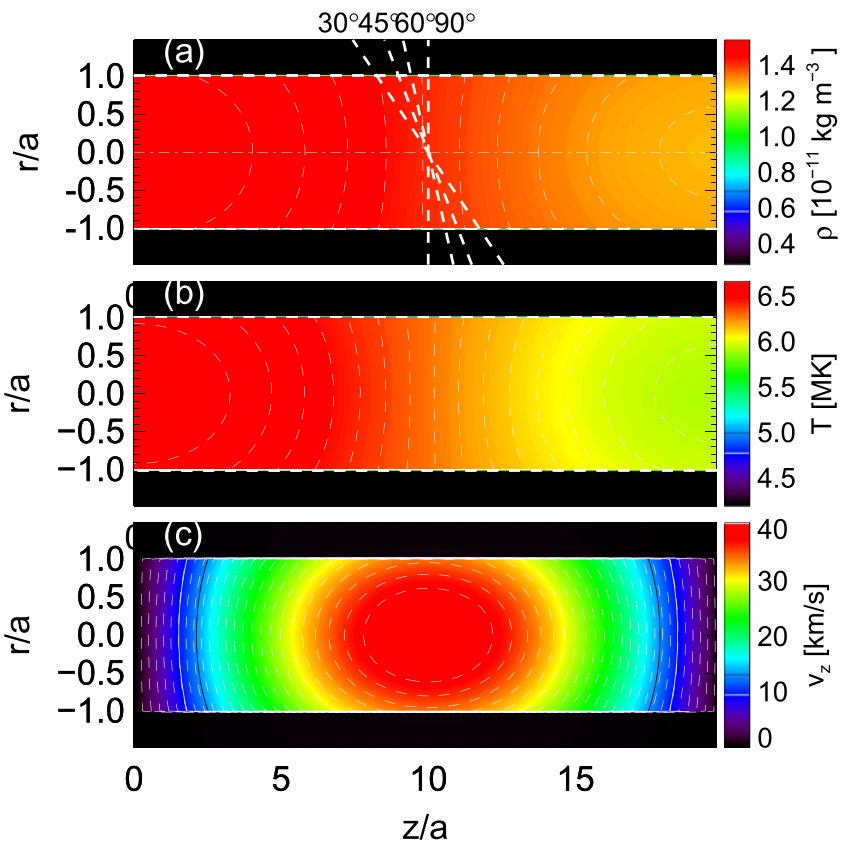

Figure 1. Snapshots of (a) $\rho(r, z)$, (b) $T(r, z)$, and (c) $v_{z}(r, z)$ at $t=P_{0} / 8$. The dashed lines indicate the position of the rays at LOS of $30^{\circ}, 45^{\circ}, 60^{\circ}$, and $90^{\circ}$, respectively.

respectively, where $k=\pi n / L_{0}$ is the longitudinal wavenumber, $n$ is the longitudinal mode number, $L_{0}$ is the length of the loop, and $\gamma=5 / 3$ is the adiabatic index.

Equations (2)-(5) are solved in cylindrical coordinates $(r, \phi, z)$ with the boundary condition at $r=a$, where the radial displacement $\xi_{r}$ and the total pressure are kept in balance. In the case of the standing slow mode with $m=0$, we Fourier-analyze the perturbed quantities by assuming $P_{\mathrm{T} 1}=A \mathcal{R}(r) \cos (\omega t) \cos (k z)$, where $A$ is the amplitude of the perturbed total pressure and $\mathcal{R}$ is defined as

$$
\mathcal{R}=\frac{\omega^{2}}{\rho_{0}\left(C_{\mathrm{s}}^{2}+C_{\mathrm{A}}^{2}\right)\left(\omega^{2}-\omega_{\mathrm{T}}^{2}\right)} \frac{d}{d t} \mathcal{R}^{\prime}
$$

Here $\mathcal{R}$ ' is the relevant " $\mathcal{R}$ " in Antolin \& Van Doorsselaere (2013). We use a $\cos (k z)$ longitudinal profile in total pressure perturbation, so the density (temperature) nodes are fixed at footpoints, while the longitudinal velocity perturbation follows a profile of $\sin (\mathrm{kz})$ and thus has a node at the loop apex for the fundamental mode. A key derivation is that

$$
\nabla \cdot \boldsymbol{\xi}=-\frac{\omega^{2} P_{\mathrm{T} 1}}{\rho_{0}\left(C_{\mathrm{s}}^{2}+C_{\mathrm{A}}^{2}\right)\left(\omega^{2}-\omega_{\mathrm{T}}^{2}\right)} .
$$

The perturbed total pressure must satisfy

$$
\frac{d^{2} P_{\mathrm{T} 1}}{d r^{2}}+\frac{d P_{\mathrm{T} 1}}{r d r}-\kappa_{r}^{2} P_{\mathrm{T} 1}=0
$$

where $\kappa_{r}^{2}=\frac{\left(\omega_{\mathrm{s}}^{2}-\omega^{2}\right)\left(\omega_{\mathrm{A}}^{2}-\omega^{2}\right)}{\left(\omega_{\mathrm{s}}^{2}+\omega_{\mathrm{A}}^{2}\right)\left(\omega_{\mathrm{T}}^{2}-\omega^{2}\right)} k^{2}$ is a modified radial wavenumber and has the dimensionality of wavenumber $k$. Equation (8) holds for both internal and external plasmas, where all quantities are piecewise functions of $r$ (Figure 1). Equation (8) gives

$$
\mathcal{R}=\left\{\begin{array}{cc}
J_{0}\left(\left|\kappa_{r \mathrm{i}}\right| r\right) & : r \leqslant a \\
K_{0}\left(\kappa_{r \mathrm{e}} r\right) & : r>a .
\end{array}\right.
$$

By matching the boundary conditions, we obtain the dispersion relation for the fast and slow sausage body mode (Edwin \& Roberts $1983 ; \kappa_{r \mathrm{e}}^{2}>0$ and $\kappa_{r \mathrm{i}}^{2}<0$, and hence we redefine

$$
\begin{aligned}
& \left.\left|\kappa_{r \mathrm{i}}\right|=\sqrt{-\kappa_{r \mathrm{i}}^{2}}\right) \\
& \quad \frac{\kappa_{r \mathrm{e}}}{\rho_{\mathrm{e}}\left(\omega_{\mathrm{Ae}}^{2}-\omega^{2}\right)} \frac{K_{0}^{\prime}\left(\kappa_{r \mathrm{e}} a\right)}{K_{0}\left(\kappa_{r \mathrm{e}} a\right)}=\frac{\kappa_{r \mathrm{i}}}{\rho_{\mathrm{i}}\left(\omega_{\mathrm{Ai}}^{2}-\omega^{2}\right)} \frac{J_{0}^{\prime}\left(\left|\kappa_{r \mathrm{i}}\right| a\right)}{J_{0}\left(\left|\kappa_{r \mathrm{i}}\right| a\right)},
\end{aligned}
$$

where $J_{0}$ is the zeroth-order Bessel function of the first kind and $K_{0}$ is the zeroth-order modified Bessel functions of the second kind; $J_{0}^{\prime}$ and $K_{0}^{\prime}$ are the corresponding derivatives with respect to $\kappa_{r} r$. The perturbed thermodynamic quantities, which affect plasma emission, are the velocity vector $\boldsymbol{v}=\partial \boldsymbol{\xi} / \partial t$, the plasma density $\rho$, and the temperature $T$ :

$$
\begin{gathered}
v_{r}=-\frac{A d \mathcal{R} / d r \omega}{\rho_{0}\left(\omega^{2}-\omega_{\mathrm{A}}^{2}\right)} \sin (\omega t) \cos (k z), \\
v_{z}=-\frac{A \mathcal{R} C_{\mathrm{T}}^{2} k \omega}{\rho_{0} V_{\mathrm{A}}^{2}\left(\omega^{2}-\omega_{\mathrm{T}}^{2}\right)} \sin (\omega t) \sin (k z), \\
\rho_{1}=\frac{A \mathcal{R}}{\left(C_{\mathrm{s}}^{2}+C_{\mathrm{A}}^{2}\right)} \frac{\omega^{2}}{\left(\omega^{2}-\omega_{\mathrm{T}}^{2}\right)} \cos (\omega t) \cos (k z), \\
T_{1}=\frac{A \mathcal{R}(\gamma-1) T_{0}}{\rho_{0}\left(C_{\mathrm{s}}^{2}+C_{\mathrm{A}}^{2}\right)} \frac{\omega^{2}}{\left(\omega^{2}-\omega_{\mathrm{T}}^{2}\right)} \cos (\omega t) \cos (k z),
\end{gathered}
$$

where $T_{0}$ is the equilibrium plasma temperature. The thermodynamic variables are related by the equation of state for fully ionized hydrogen $p=2 k_{\mathrm{B}} \rho T / m_{\mathrm{p}}$, where $\rho=\rho_{0}+\rho_{1}$, $T=T_{0}+T_{1}, \quad k_{\mathrm{B}}$ is the Boltzmann constant, and $m_{\mathrm{p}}$ is proton mass.

Equations (8)-(15) hold for both the fast and slow sausage mode. The solution depends on whether the equations are solved in the Alfvén $\left[\omega_{\mathrm{Ai}}, \omega_{\mathrm{Ae}}\right]$ or acoustic $\left[\omega_{\mathrm{Ti}}, \omega_{\mathrm{si}}\right]$ frequency range (Sakurai et al. 1991). We note that $v_{r} / v_{z} \propto$ $\left(\omega^{2}-\omega_{\mathrm{T}}^{2}\right) /\left(\omega^{2}-\omega_{\mathrm{A}}^{2}\right)$. Therefore, in the Alfvén frequency range, $v_{r} / v_{z} \gg 1$, it corresponds to the fast sausage mode (Antolin \& Van Doorsselaere 2013; Reznikova et al. 2014), while in the acoustic frequency range, $v_{r} / v_{z} \ll 1$, this corresponds to the slow sausage mode (see, e.g., Moreels \& Van Doorsselaere 2013), which is the topic of this study.

\subsection{Hot Flaring Coronal Loop}

Hot coronal loops are complex and highly dynamic structures heated to $2-30 \mathrm{MK}$ by flares (see a review by Reale 2014). A coronal loop may have unresolved fine structures (Priest et al. 2002; Van Doorsselaere et al. 2014) or multiple strands (Antolin \& Rouppe van der Voort 2012; Peter et al. 2013; Antolin et al. 2014, 2015; Scullion et al. 2014). Heating/cooling (see, e.g., Klimchuk 2006; Hood et al. 2009) and the associated flows (see Winebarger et al. 2002) are usually detected at the footpoints of the loops. 
MHD wave theory (see, e.g., Edwin \& Roberts 1983; Sakurai et al. 1991; Goossens et al. 2011) normally assumes that a quiescent loop is in equilibrium with the ambient plasma, and therefore the heating and cooling timescales should be sufficiently longer than the MHD timescales (in order of minutes in the case of slow modes). If a loop is subject to active heating or cooling, the Wentzel-Kramers-Brillouin (WKB) approximation can be used (similar to Ruderman 2011a, 2011b).

In this study, we are only concerned with the observational features of established standing slow modes; even if the WKB approximation is violated, i.e., the MHD slow-mode timescale is on the same order of the heating or cooling timescale, the result could still be used to identify a standing slow wave based on stepwisely defined quasi-equilibriums. We set up a hot and dense flaring coronal loop with typical parameters that are observed by SUMER (Wang 2011). Our loop measures $L_{0}=100 \mathrm{Mm}$ in length and $a=5 \mathrm{Mm}$ in radius. The loop is filled with plasma with a density $\rho_{0}=1.4 \cdot 10^{-11} \mathrm{~kg} \mathrm{~m}^{-3}$ (the electron density $n_{\mathrm{e} 0}=8.5 \cdot 10^{9} \mathrm{~cm}^{-3}$ ) and a temperature of $T_{\mathrm{i}}=6.4 \mathrm{MK}$. The internal magnetic field is fixed at $B_{\mathrm{i}}=40 \mathrm{G}$. We choose a density ratio of $\rho_{\mathrm{i}} / \rho_{\mathrm{e}}=5$, a temperature ratio $T_{\mathrm{i}} / T_{\mathrm{e}}=1.5$, and a magnetic field strength ratio $B_{\mathrm{i}} / B_{\mathrm{e}}=0.91$. The ratios of the plasma parameters are set in the typical range of flaring coronal loops. Changes in these ratios will not significantly affect the results, since in a slow mode the longitudinal perturbations are strictly confined within the plasma cylinder and are more than four orders of magnitude stronger than the perturbations to the ambient plasma. In this setup, the internal and external plasmas are typical coronal fluids with plasma beta of $\beta_{\mathrm{i}}=0.23$ and $\beta_{\mathrm{e}}=0.026$, respectively. The acoustic speeds are $C_{\mathrm{si}}=420 \mathrm{~km} \mathrm{~s}^{-1}$ and $C_{\mathrm{se}}=340 \mathrm{~km} \mathrm{~s}^{-1}$, while the Alfvén speeds are $C_{\mathrm{Ai}}=950 \mathrm{~km} \mathrm{~s}^{-1}$ and $C_{\mathrm{Ae}}=2300 \mathrm{~km} \mathrm{~s}^{-1}$ (Table 1). These speeds are typical values observed in the solar corona (see, e.g., Aschwanden 2005). We also investigate the slow modes in hot loops at $T_{\mathrm{i}}=8.8,12,15,20 \mathrm{MK}$ (see Table 1) and explore how the properties would deviate from the case of the $6.4 \mathrm{MK}$ loop. The total pressure perturbation is kept unchanged; the amplitude of perturbed density, temperature, and velocity will vary according to the equilibrium temperature (Table 1). However, the ampltiude of the perturbed quantities will not affect the overall result, since in linearized MHD wave modes they are scalable. In the following text, we refer to the case of slow wave in the $6.4 \mathrm{MK}$ loop by default, and other cases are specified as otherwise in Section 4.3.

For $n=1$ (the fundamental longitudinal mode), $k a=0.157$ is in the long-wavelength limit. We use $A_{\mathrm{i}}=0.01$ so that the velocity perturbation is about $57 \mathrm{~km} \mathrm{~s}^{-1}$ and the density perturbation is about $12 \%$ of the equilibrium value. For $n=2(3)$, we use $A_{\mathrm{i}}=0.02(0.04)$. The velocity and density perturbation are about $41(48) \mathrm{km} \mathrm{s}^{-1}$ and $9(11) \%$ of the equilibrium value, respectively (Table 1). These amplitude values are chosen to agree with the observed Doppler shift velocities in Wang (2011). By solving the dispersion relation Equation (10), we obtained the periods $P_{0}=520,260,170 \mathrm{~s}$ for the $n=1,2,3$ modes, respectively.

Starting from the equilibrium loop model, we construct a discrete standing slow-wave model as specified by Equations (11)-(15). The simulation domain ranges are $[0,1.5 a],[0,2 \pi]$, and $\left[0, L_{0}\right]$ for $r$-, $\phi$-, and $z$-axes, with grid cells of $150 \times 180 \times 300$, respectively. Figure 1 illustrates a snapshot of the $\rho, T$, and $v_{z}$ distribution at $t=P_{0} / 8$ for the $n=1$ mode. An antinode in terms of density perturbation is present at $z=L_{0} / 2$; this is in agreement with SUMER observations that Doppler shift oscillations are usually effectively detected at the loop apex rather than the loop footpoints (Wang et al. 2007). The density and temperature perturbations are in phase and are a quarter period out of phase, with respect to both time and space, with the longitudinal velocity. We note that the density/temperature and velocity perturbations are in phase for propagating slow waves (Sakurai et al. 2002; Wang et al. 2009b); therefore, a mix of propagating and standing wave would lead to the detection of a rather complex phase lag (Wang et al. 2009a). In this study, we focus on purely standing slow MHD waves to obtain guidelines for observations.

\section{METHODS}

\subsection{Spectroscopic Modeling}

We are concerned with synthesizing the EUV emission intensity $I_{\lambda_{0}}\left(\mathrm{ergs} \mathrm{cm} \mathrm{cm}^{-2} \mathrm{~s}^{-1} \mathrm{sr}^{-1}\right)$ of a specific spectral line $\lambda_{0}$ for optically thin plasma along the LOS (Dere et al. 1997),

$$
I_{\lambda_{0}}=\frac{A_{\mathrm{b}}}{4 \pi} \int G_{\lambda_{0}}\left(n_{\mathrm{e}}, T\right) n_{\mathrm{e}}^{2} d l
$$

where $A_{\mathrm{b}}$ is the abundance of the emitting element relative to hydrogen, $G_{\lambda_{0}}\left(\operatorname{ergs~} \mathrm{cm}^{3} \mathrm{~s}^{-1}\right)$ is the contribution function that contains the terms relative to atomic physics, and $d l$ is an infinitesimal element length along the LOS.

To calculate the integrated emission intensity in Equation (16), we generate a look-up table for the Fe XIX $\lambda 1118.1$ line using the CHIANTI v7.1 atomic database (Dere et al. 1997; Landi et al. 2013). The look-up table is sampled at a uniform mesh of size $200 \times 200$ grid points at $\log n_{\mathrm{e}}\left(\mathrm{cm}^{-3}\right) \in[8,11]$ and $\log T(\mathrm{~K}) \in[6.2,7.7]$. We used the CHIANTI collisional ionization equilibrium file chianti v7.ioneq and coronal abundance Sun coronal 2012 schmélz.abund (also see Antolin \& Van Doorsselaere 2013$){ }^{5}$ The choices of ionization and abundance files do not affect our results at all, since we are only concerned with the relative intensity perturbation and Doppler shift caused by the MHD waves.

Figure 2 illustrates the forward modeling method and how the LOS integration (Equation (16)) is implemented numerically. For each grid point, the emissivity $\epsilon^{\lambda_{0}}=G_{\lambda_{0}} n_{\mathrm{e}}^{2}$ is calculated and is spread to a Gaussian spectrum with the width determined by the thermal broadening of the spectral line. The spectrum covered in this study is centered at $1118.1 \AA$ with a range of $\pm 0.5 \AA\left( \pm 130 \mathrm{~km} \mathrm{~s}^{-1}\right)$. This is an Fe XIX EUV line, in which most spectroscopic observations on standing slow wave were performed (see Wang 2011). We used 60 wavelength values to sample the spectrum. This corresponds to a spectral resolution of $\Delta \lambda=16.7 \mathrm{m \AA}$ or $\Delta v_{\mathrm{D}}=4.5 \mathrm{~km} \mathrm{~s}^{-1}$, which is sufficient to resolve the spectrum. Then along the LOS, the emission wavelength of the elementary plasma fluid is modified by the velocity perturbation caused by the wave and is rebinned into the discrete spectrum. By assuming a Gaussian distribution for the integrated spectrum, we obtain the emission intensity $I_{\lambda_{0}}$ and Doppler shift velocity $v_{\mathrm{D}}$.

\footnotetext{
5 The source of the forward modeling code (FoMo) is available at https:// wiki.esat.kuleuven.be/FoMo.
} 
Table 1

Parameters of the Loop Systems and the Standing Slow Modes

\begin{tabular}{|c|c|c|c|c|c|}
\hline Loops & $T_{\mathrm{i}}=6.4 \mathrm{MK}$ & $T_{\mathrm{i}}=8.8 \mathrm{MK}$ & $T_{\mathrm{i}}=12 \mathrm{MK}$ & $T_{\mathrm{i}}=15 \mathrm{MK}$ & $T_{\mathrm{i}}=20 \mathrm{MK}$ \\
\hline$L_{0}(\mathrm{Mm})$ & 100 & 100 & 100 & 100 & 100 \\
\hline$a(\mathrm{Mm})$ & 5.0 & 5.0 & 5.0 & 5.0 & 5.0 \\
\hline$B_{\mathrm{e}}(\mathrm{G})$ & 43.8 & 45.3 & 47.0 & 48.7 & 51.6 \\
\hline$\rho_{\mathrm{i}}\left(10^{-11} \mathrm{~kg} \mathrm{~m}^{-3}\right)$ & 1.4 & 1.4 & 1.4 & 1.4 & 1.4 \\
\hline$n_{e \mathrm{i}}\left(10^{9} \mathrm{~cm}^{-3}\right)$ & 8.5 & 8.5 & 8.5 & 8.5 & 8.5 \\
\hline$T_{\mathrm{i}} / T_{\mathrm{e}}$ & 1.5 & 1.5 & 1.5 & 1.5 & 2.0 \\
\hline$\beta_{\mathrm{i}}$ & 0.23 & 0.32 & 0.44 & 0.55 & 0.74 \\
\hline$\beta_{\mathrm{e}}$ & 0.026 & 0.034 & 0.043 & 0.050 & 0.044 \\
\hline$C_{\mathrm{si}}\left(\mathrm{km} \mathrm{s}^{-1}\right)$ & 420 & 490 & 570 & 640 & 740 \\
\hline$V_{\mathrm{Ai}}\left(\mathrm{km} \mathrm{s}^{-1}\right)$ & 950 & 950 & 950 & 950 & 950 \\
\hline & & & $n=1, A=0.01$ & & \\
\hline$v_{z}^{0}\left(\mathrm{~km} \mathrm{~s}^{-1}\right)^{\mathrm{a}}$ & 57 & 47 & 38 & 31 & 28 \\
\hline$\rho_{1}^{0} / \rho_{\mathrm{i}}$ & 0.12 & 0.08 & 0.06 & 0.04 & 0.03 \\
\hline \multirow[t]{2}{*}{$T_{1}^{0} / T_{\mathrm{i}}$} & 0.08 & 0.06 & 0.04 & 0.03 & 0.02 \\
\hline & & & $n=2, A=0.02$ & & \\
\hline$v_{z}^{0}\left(\mathrm{~km} \mathrm{~s}^{-1}\right)$ & 41 & $\ldots$ & $\ldots$ & $\ldots$ & $\ldots$ \\
\hline$\rho_{1}^{0} / \rho_{\mathrm{i}}$ & 0.09 & $\cdots$ & $\cdots$ & $\cdots$ & $\cdots$ \\
\hline \multirow[t]{2}{*}{$T_{1}^{0} / T_{\mathrm{i}}$} & 0.06 & $\cdots$ & $\cdots$ & $\cdots$ & $\cdots$ \\
\hline & & & $n=3, A=0.04$ & & \\
\hline$v_{z}^{0}\left(\mathrm{~km} \mathrm{~s}^{-1}\right)$ & 48 & $\cdots$ & $\cdots$ & $\ldots$ & $\ldots$ \\
\hline$\rho_{1}^{0} / \rho_{\mathrm{i}}$ & 0.11 & $\ldots$ & $\ldots$ & $\cdots$ & $\ldots$ \\
\hline
\end{tabular}

Note.

a The superscript 0 here and hereafter denotes the amplitude of the perturbed quantities in Equations (11)-(15), excluding the spatial and temporal terms.

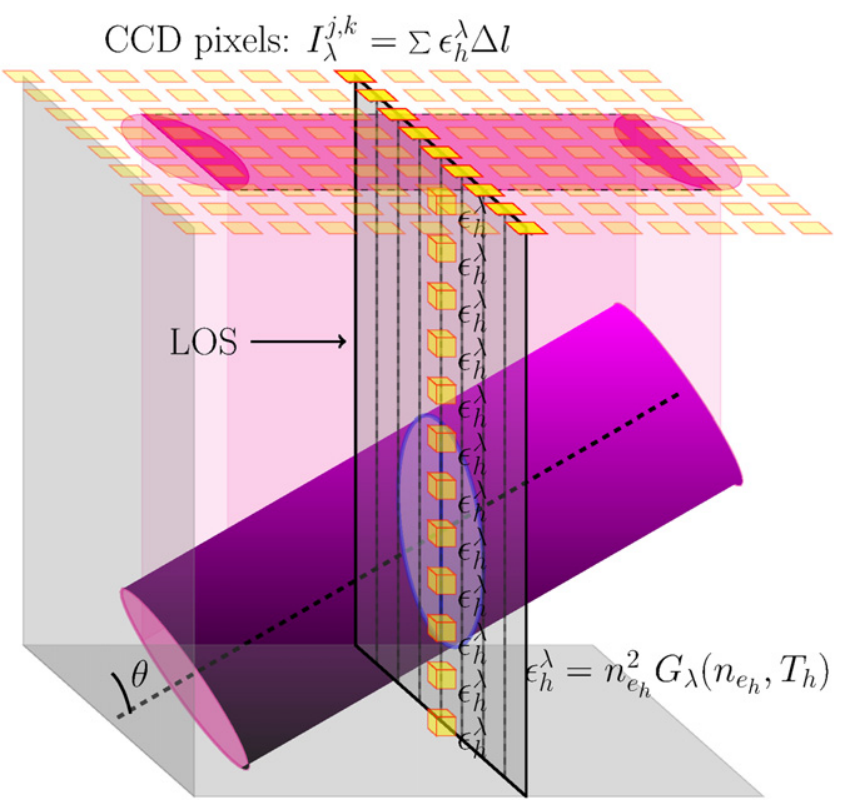

Figure 2. Schematic diagram illustrating the forward modeling method and LOS integrations.
We perform the calculations for LOS angles $\theta=30^{\circ}, 45^{\circ}, 60^{\circ}$, and $90^{\circ}$, respectively. The projected plane of sky has a mesh grid of $N_{x} \times N_{z}=45 \times 300 \sin \theta$, so that the synthesized LOS emission plane has a pixel size of $0.33 \times 0.33 \mathrm{Mm}^{2}$ (Figure 2).

\subsection{Imaging Modeling}

To synthesize the observational features of SDO/AIA channels, we calculated the AIA temperature response function $K_{\alpha}\left(n_{\mathrm{e}}, T\right)\left(\mathrm{DN} \mathrm{cm}^{5} \mathrm{~s}^{-1}\right)$ for bandpass $\alpha$ (Boerner et al. 2012):

$$
K_{\alpha}\left(n_{\mathrm{e}}, T\right)=\int_{0}^{\infty} G\left(\lambda, n_{\mathrm{e}}, T\right) R_{\alpha}(\lambda) d \lambda,
$$

where $R_{\alpha}(\lambda)\left(\mathrm{DN} \mathrm{cm}^{2}\right.$ sr photon $\left.{ }^{-1}\right)$ is the instrument-wavelength response function. This is the product of the solid angle occupied by a unit surface $\left(0\right.$ ". $\left.6 \times 0^{\prime \prime} .6\right)$ relative to the telescope and the value calculated with the aia_get_resp.pro. $G\left(\lambda, n_{\mathrm{e}}, T\right)$ (photon $\mathrm{cm}^{3} \mathrm{~s}^{-1} \mathrm{sr}^{-1}$ ) is the contribution function calculated with the isothermal.pro routine in CHIANTI (see Del Zanna et al. 2011). Then the flux $F_{\alpha}(\boldsymbol{x})\left(\mathrm{DN} \mathrm{s}^{-1}\right)$ at pixel $\boldsymbol{x}$ is integrated along the LOS,

$$
F_{\alpha}(\boldsymbol{x})=\int_{l} K_{\alpha}\left(n_{\mathrm{e}}, T\right) n_{\mathrm{e}}^{2} d l .
$$



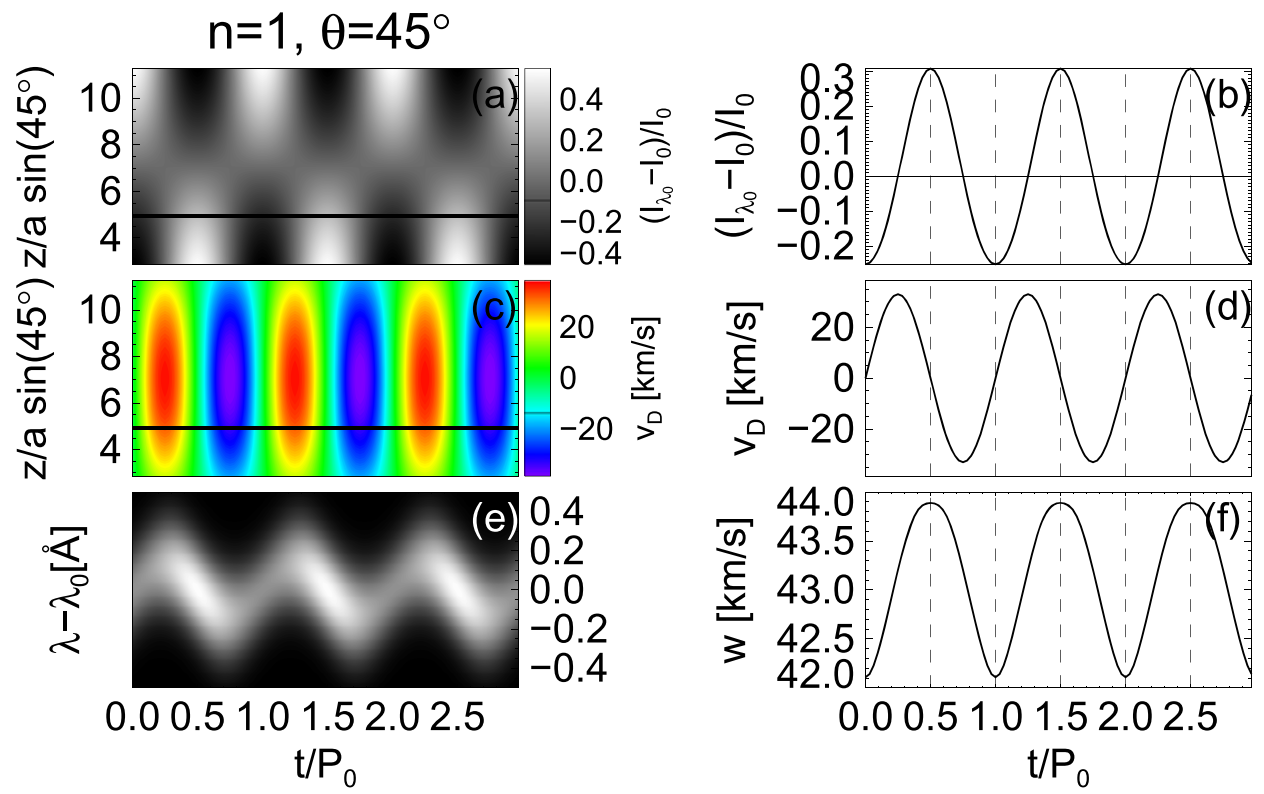

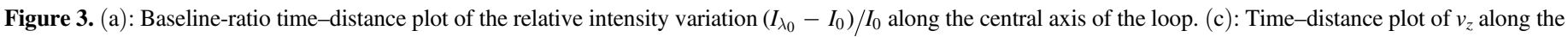

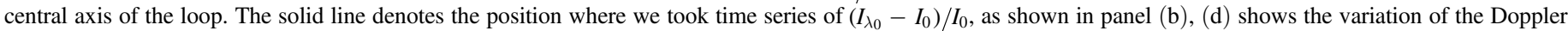

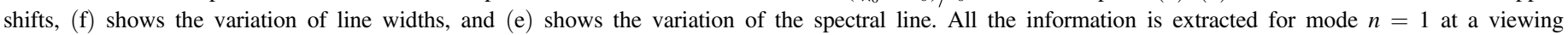
angle $\theta=45^{\circ}$.

Equation (18) is solved using the same algorithm as Equation (16) (Figure 2); however, the intensity is obtained by summing up the contributing emissions in all effective wavelengths (Equation (17)) rather than spreading into a spectrum (see Section 3.1). A look-up table for each AIA bandpass is sampled at a uniform mesh of size $200 \times 200$ grid points at $\log n_{\mathrm{e}}\left(\mathrm{cm}^{-3}\right) \in[8,11]$ and $\log T(\mathrm{~K}) \in[4,8]$.

We synthesized the AIA $94 \AA$ channel that would image our flaring loops $(>6.4 \mathrm{MK})$. The resultant pixel size is kept uniform at 0 ". $6 \times 0$ ". 6 ; therefore, we choose a mesh grid of $N_{x} \times N_{z}=35 \times 230 \sin \theta$ for output and perform the calculations for LOS angles $\theta=30^{\circ}, 45^{\circ}, 60^{\circ}$, and $90^{\circ}$, respectively. Point-spread functions (PSFs; see Antolin \& Van Doorsselaere 2013; Poduval et al. 2013) would only have marginal effect at the edges and the cylinder boundaries. Moreover, we use long-wavelength limits, and the plasma motions are predominantly longitudinal; thus, PSF effect is neglected in this study.

\section{RESULTS}

In this section, we divide the results into two categories that observers mainly use to detect standing slow mode in a coronal loop. As we use linearization of the MHD wave model and perform geometric integration by modeling the atomic emissions, the results could be scaled to the range of interests. In the following subsections, we present a typical observation mode of a spectrograph or imager and study other effects that may affect the observations.

\subsection{Spectroscopic Observations \\ 4.1.1. Typical Observation}

We first mimic a sit-and-stare campaign of a spectrograph, e.g., SUMER. The slit is ideally placed to fully cover the central line of our loop with a viewing angle of $45^{\circ}$. Figure 3 presents typical observables of a spectrograph. The intensity perturbation (Figure 3(a)) has a larger value at the loop footpoints than at the loop apex, while the Doppler shift $v_{\mathrm{D}}$ (Figure 3(c)) shows an opposite spatial pattern: it has a maximum at the loop apex. This is in agreement with the standing slow-wave model (see Section 2.2), in which the longitudinal velocity and density are phase-shifted in space by a quarter wavelength. Time series (Figures 3(b) and (d)) taken at a position off the loop apex and footpoints show a typical observation, which could be directly compared with Figure 2 in Wang (2011): the intensity perturbation oscillates with a quarter period out of phase in time with the Doppler shift $v_{\mathrm{D}}$. We also show that intensity variations are in phase with line width $w$ oscillations (Figures 3(b) and (f)). Figure 3(e) illustrates a typical spectrum that would be observed in a sitand-stare mode: the spectral line is Doppler-shifted by the standing slow wave and is also broadened by the perturbed velocity and temperature along LOS. Spectral observations at a slit position could provide the first signal of an MHD wave. The line width variation is not reported so far by any observations; its temporal variation and phase relations with other observables could be used to identify the wave mode. This may be due to the low amplitude of the line width oscillation (about $1 \mathrm{~km} \mathrm{~s}^{-1}$ ); thus, it is beyond the detection capability of current instruments.

When the viewing angle is normal to the loop, i.e., $90^{\circ}$ (Figure 4), the intensity and line width broadening modulation are still significant and are in phase with each other. However, the Doppler shift $v_{\mathrm{D}}$ oscillation becomes below noise level and is not detectable. If a slit is placed over the loop apex (antinode), then the intensity and line width modulation will be very small.

A measure of goodness-of-fit $\chi^{2}$ to a Gaussian spectrum is not investigated in this study (see, e.g., Antolin \& Van Doorsselaere 2013). It measures the level of goodness that a 


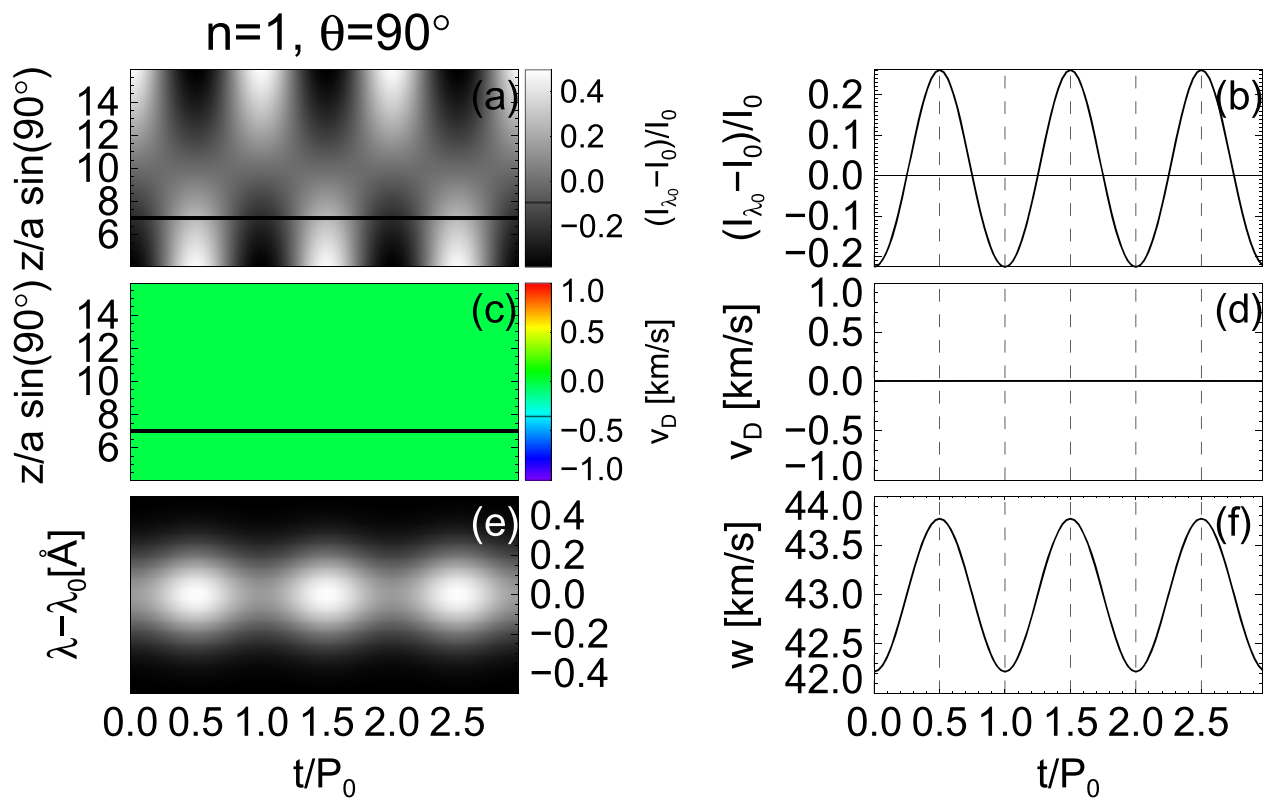

Figure 4. Same as Figure 3, but for $n=1, \theta=90^{\circ}$.
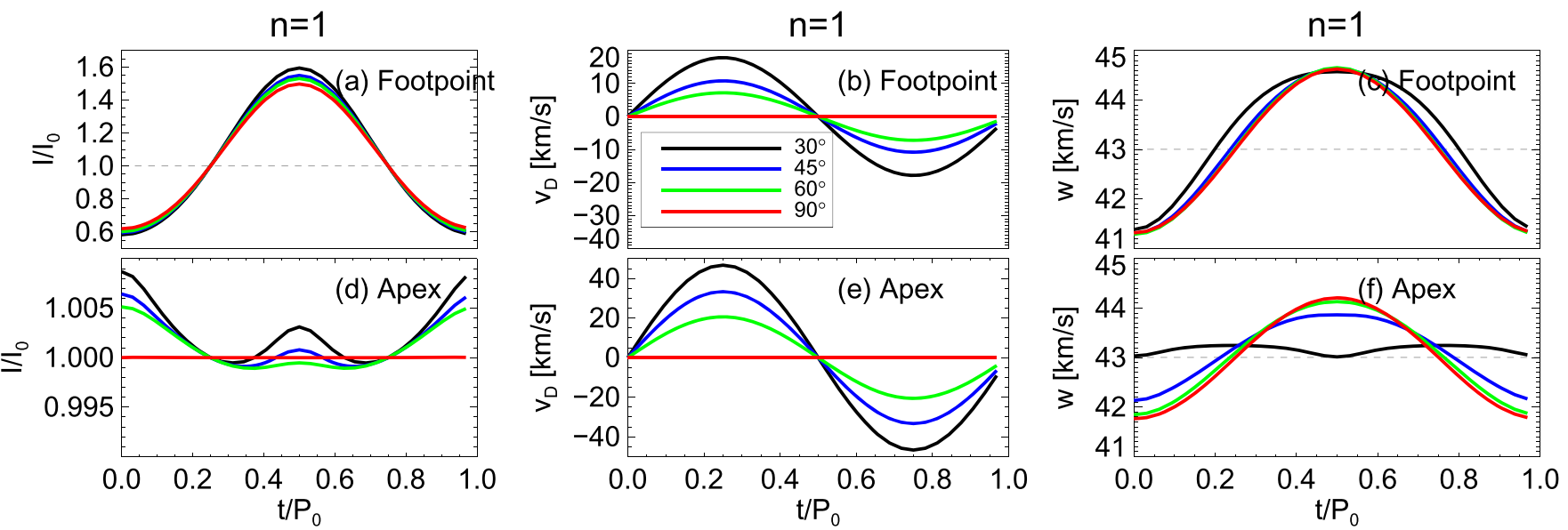

Figure 5. One period variation of the normalized emission intensity $I / I_{0}(\mathrm{a}, \mathrm{d})$, Doppler shift velocity $v_{\mathrm{D}}(\mathrm{b}, \mathrm{e})$, and line width $w(\mathrm{c}, \mathrm{f})$ observed at the loop footpoint and apex for the $n=1$ mode.

combination of multiple-temperature spectral components could approximate a single Gaussian spectrum. For the standing slow mode, the plasma motions are predominately longitudinal, so plasma advections across the cylinder boundary are negligible. Therefore, the spectra rarely deviate from a Gaussian shape. In our study, the $\chi^{2}$ measures at the order of $10^{-4}$. This means that the Gaussian profiles are in accord with the spectra or the error variance has been overestimated. The latter is true in our case, since we do not have error bars associated with the spectra and unity is used as error variance.

\subsubsection{LOS Effect}

Figure 5 presents the two extreme cases at the loop apex and footpoints. ${ }^{6}$ At the footpoints, the intensity and line width

\footnotetext{
6 We truncated $2 a / \sin 30^{\circ}=4 a$ off the loop ends, where rays with an LOS angle of $30^{\circ}$ would traverse only part of the loop cross section and will contain an edge effect; therefore, we refer to the positions of $z=4 a, L_{0}-4 a$ as footpoints.
}

variations do not change significantly with varying LOS angles; this is because the wavelength of $n=1$ standing slow mode is significantly longer than the loop radius. Thus, rays of any LOS angle are less likely to traverse both the node and antinode. This is contrary to the short-wavelength case for the fast sausage mode (Antolin \& Van Doorsselaere 2013). In the short-wavelength limit, LOS rays would traverse more fine structures along both radial and longitudinal directions; therefore, the intensity and line width modulations are more complicated. However, in our case, the modulation of Doppler shift is significantly affected by LOS angles, since the longitudinal velocity dominates the perturbation. In a rough approximation, the amplitude of Doppler shift could be linearly de-projected by a factor of $1-\theta / 90^{\circ}$ or $1-2 \theta / \pi$, rather than $\cos \theta$ (Figure 6). A $\cos \theta$ de-projection could overestimate the velocity perturbation by a factor of more than $10 \%$. This empirical formula could be used in observations. The reason for the deviation from simple geometric projection is that each fluid element is projected by $\cos \theta$, while the overall Dopplershifted spectrum including the contribution of all fluid elements 


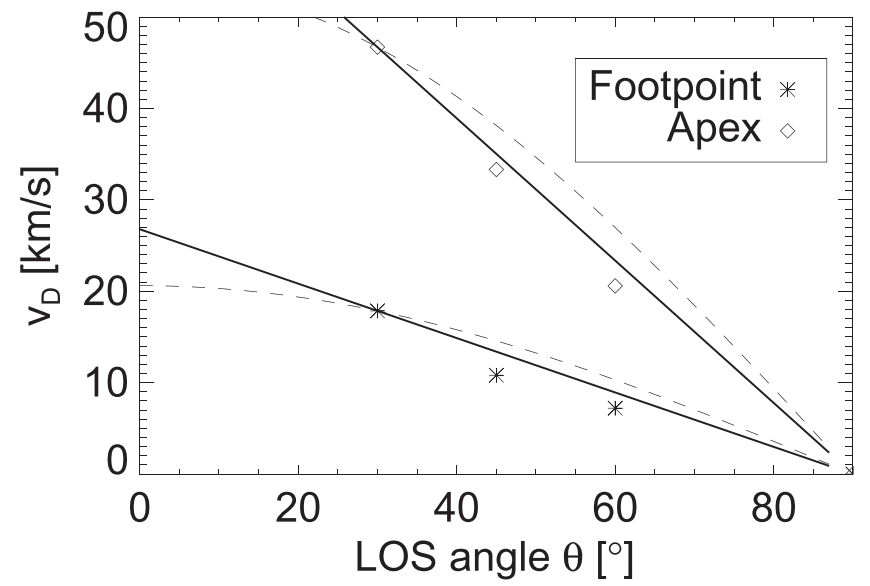

Figure 6. Doppler shift velocity amplitude as a function of LOS viewing angle at the footpoint (asterisks) and apex (diamonds). The dashed and solid lines represent the trend of $\cos \theta$ and $(1-\theta / 90)$ dependence, respectively.

along LOS does not necessarily follow the same trend. This result will affect the estimate of realistic longitudinal velocity perturbation with a two-dimensional observation and the associated wave energy budget. The LOS effect may also lead to incorrect longitudinal mode identification (see, e.g., Antolin \& Verwichte 2011).

The Doppler shift modulation exhibits similar patterns owing to LOS variation at both the apex and footpoints (Figures 5(b) and (e)). Intriguingly, we found that positive modulation of the intensity is overwhelmingly in excess over negative modulations at every LOS angle (Figures 3, 4, and 5(a)). This effect may halve the periodicity at regions where the intensity modulation is relatively weak, e.g., at the apex (Figure 4(d)). This is not introduced by asymmetry in the geometry or the distribution of electron density $n_{\mathrm{e}}$ or plasma temperature $T$. Figures 7 presents the contribution function of the Fe XIX $\lambda 1118.1$ line; the plus sign indicates the loop parameters. $G_{\lambda_{0}}\left(n_{\mathrm{e}}, T\right)$ varies less than $1 \%$ with the electron density $n_{\mathrm{e}}$ at high temperatures, but strongly peaks in temperature. In our case, positive temperature modulation leads to larger increase in $G_{\lambda_{0}}$ than the same amount of negative temperature modulation would do; therefore, we have excess intensity enhancement when temperature increases. This was also found, although not mentioned, in the case of intensity variation in the $193 \AA$ bandpass (Antolin \& Van Doorsselaere 2013). In contrast, this effect is missing in the $171 \AA$ bandpass, as the contribution function $G_{171}$ has an almost equal gradient with respect to $T$ in the temperature of interest; see Figure 6 in Antolin \& Van Doorsselaere (2013). This effect leads to a halving of periodicity in intensity and line width, especially in small LOS angles. It would become more significant for a loop with a temperature such that $\partial G_{\lambda_{0}}^{2} / \partial T^{2}$ reach extreme values. In such conditions, this effect would spread to broader spatial regions and larger LOS angles. We also notice that this effect would lead the asymmetry in emission intensity modulation (Figure 5(a)); however, it is highly likely to be neglected or removed by the data processing technique, e.g., detrending, running difference.

\subsubsection{Longitudinal Overtones}

We perform sit-and-stare mode observations for $n=2,3$ modes as well (figures are not shown here). In spectroscopic

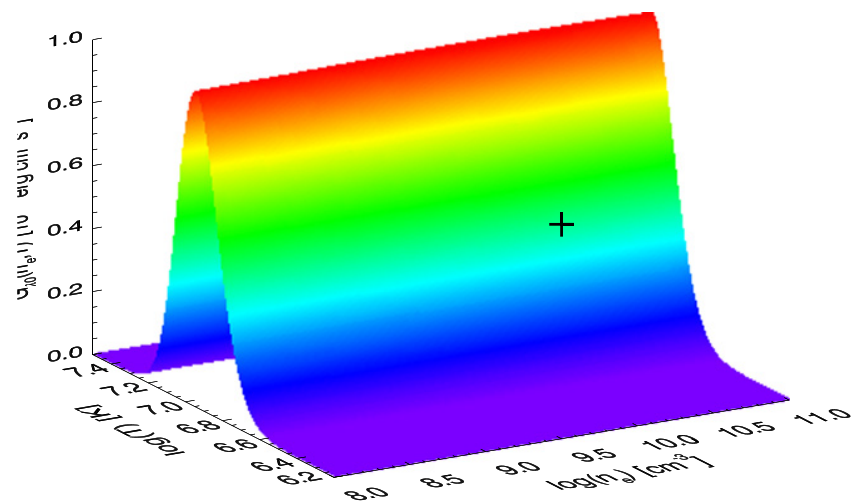

Figure 7. Contribution function $G_{\lambda_{0}}\left(n_{\mathrm{e}}, T\right)$ for the Fe XIX $\lambda 1118.1$ line. The maximum formation temperature is $\log T=6.95(T=8.9 \mathrm{MK})$. The plus sign marks the region with the used loop parameters $\left[n_{\mathrm{ei}}, T_{\mathrm{i}}\right]$.

observations, only a small segment of the spatial distribution of emission intensity and Doppler shift along a loop is normally measured. Without spatial information, it is not possible to judge the longitudinal mode number. However, with imaging observations, longitudinal overtones may be observed (see the next section).

\subsection{Imaging Observation}

In imaging observations, the spatial distribution of the emission intensity is obtained. One could easily follow the spatiotemporal variation of a loop oscillation using the timedistance method (see, e.g., Yuan \& Nakariakov 2012). Figure 8 (a) shows the baseline-ratio difference plot of $n=1$ mode along the loop. The intensity perturbation is more significant at the loop footpoints than the loop apex. By changing the viewing angle, the intensity modulation changes slightly, but the overall spatiotemporal pattern remains unchanged. This is in agreement with the spectroscopic model; see discussions in Section 4.1. The time-distance plot along a loop could reveal the nodal structures of the longitudinal overtones (Figures 8(b) and (c)). By comparing the amplitude variation along the loop, one could measure the longitudinal mode number $n$ and hence the wavelength of the slow mode $2 L_{0} / n$.

\subsection{Observing Standing Slow Mode in Hot Loops with Different Temperatures}

So far, we have only studied a $6.4 \mathrm{MK}$ loop with a fixed density and temperature ratio. As long as the background emission is insignificant compared to loop emission, the current result remains valid. However, if a coronal loop is heated to the hotter wing $\left(T_{\mathrm{i}}>8.8 \mathrm{MK}\right)$ of $G_{\lambda_{0}}$ (Figure 7$)$, the background emission could become stronger than the loop itself. In such cases, the observed Doppler shift and intensity modulation would be rather different. The free parameters are the loop temperature, density, and the temperature and density ratio. It is not practical to iterate all possible combinations, so we only present possible scenarios of how to understand a standing slow mode properly. We vary the loop temperature, while keeping other free parameters unchanged. Several loop models are set up at the various temperatures where the SUMER $\lambda 1118.1$ responses are signficant (see Table 1 ).

Figure 9 illustrates snapshots of the relative intensity, Doppler velocity, and line width of standing slow modes 
(a) $\left(I-I_{0}\right) / I_{0}, n=1$
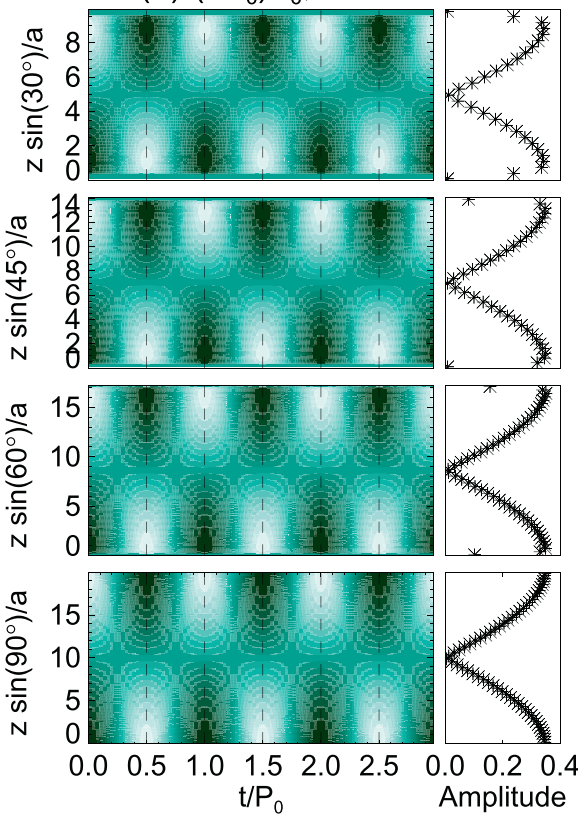

(b) $\left(I-I_{0}\right) / I_{0}, n=2$
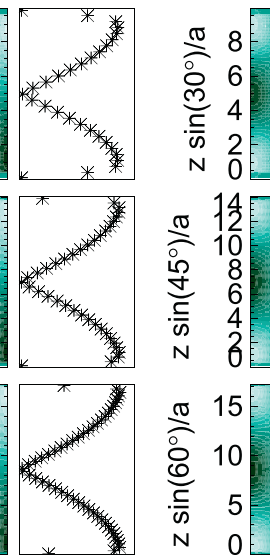

(b) $\left(1-I_{0}\right) / h_{0}$

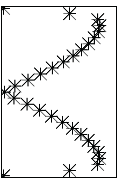

\begin{tabular}{cr} 
& 14 \\
\multirow{0}{5}{} & 10 \\
0 & 8 \\
$\frac{0}{5}$ & 6 \\
$\frac{5}{5}$ & 4 \\
N & 3
\end{tabular}
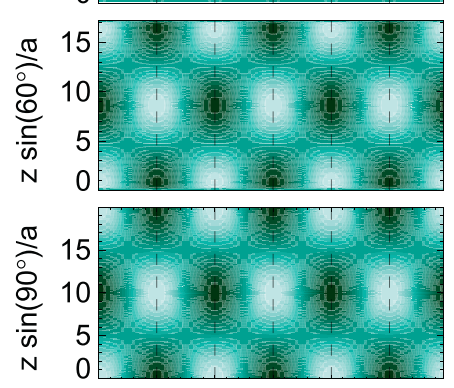

$\begin{array}{llllllllll}0.0 & 0.5 & 1.0 & 1.5 & 2.0 & 2.5 & 0.0 & 0.2 & 0.4\end{array}$ $\mathrm{t} / \mathrm{P}_{0}$
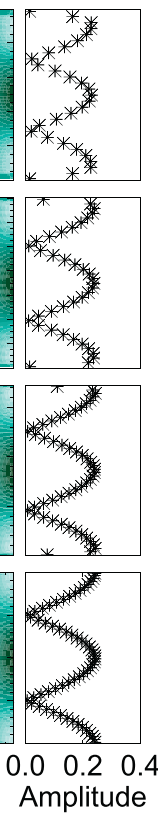

(c) $\left(I-I_{0}\right) / I_{0}, n=3$
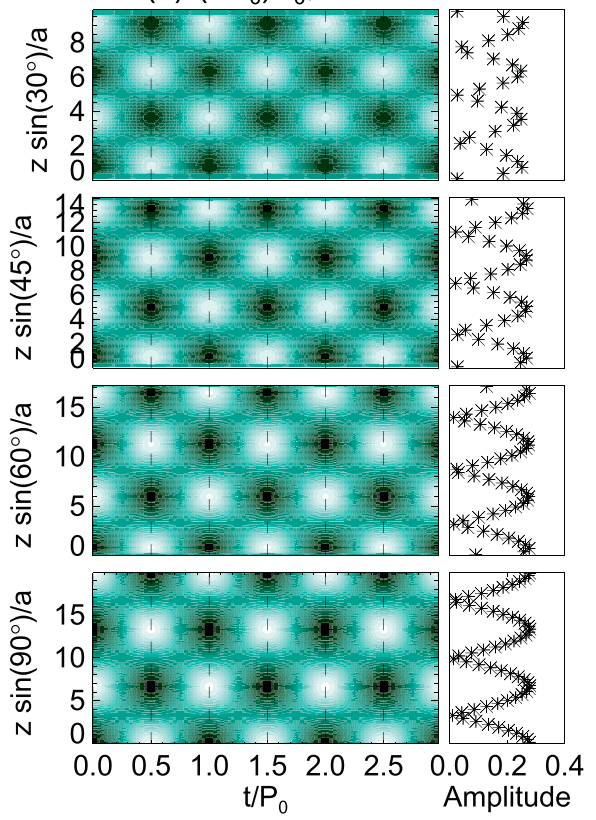

Figure 8. Baseline ratio time-distance plot of AIA $94 \AA$ emission intensity along the central axis of the loop for modes (a) $n=1$, (b) $n=2$, and (c) $n=3$.

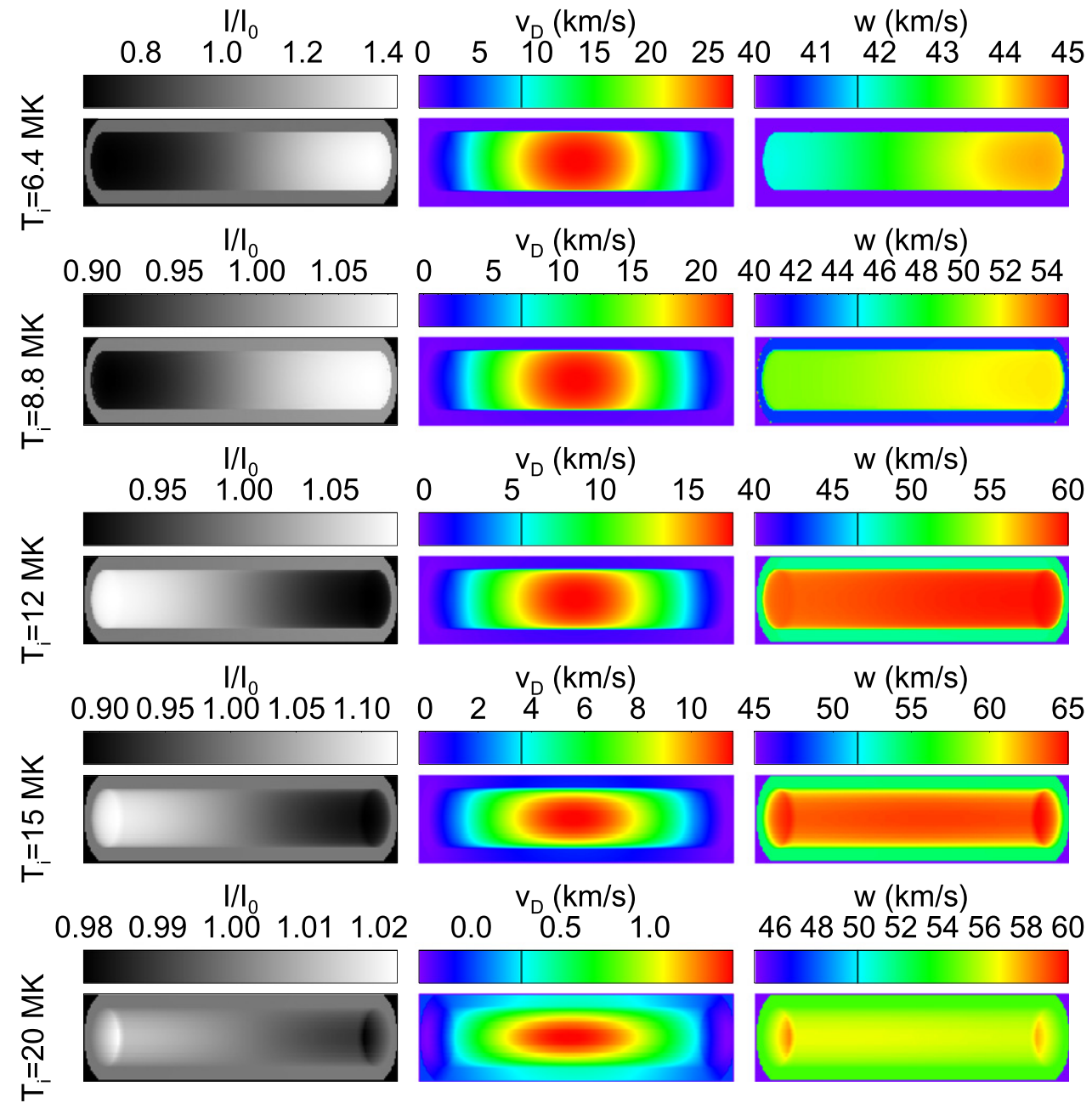

Figure 9. Snapshots $\left(\theta=45^{\circ}, t=P_{0} / 8\right)$ of the relative intensity emission (left column), the Doppler shift velocity (middle column), and the line width (right column) for loops at $T_{\mathrm{i}}=6.4,8.8,12,15,20 \mathrm{MK}$, respectively. 

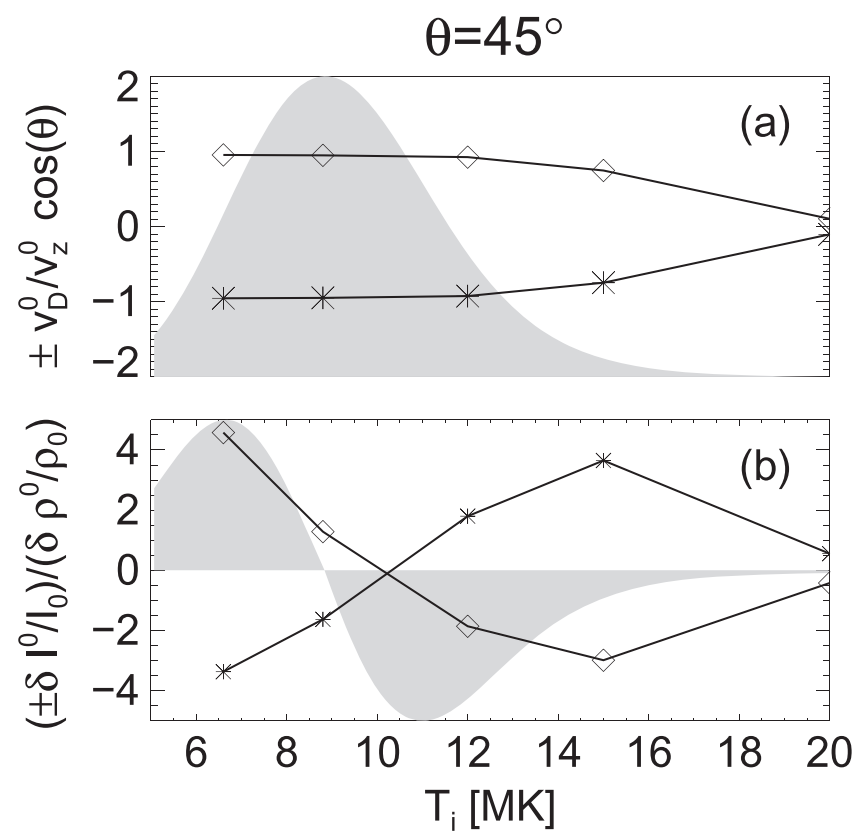

Figure 10. For loops at different temperatures, (a) presents the normalized Doppler velocity at a viewing angle of $\theta=45^{\circ}$ at loop apexes; (b) shows the normalized relative intensity modulation at the loop footpoint, where $\delta I^{0} / I_{0}=\left(I_{t=0}-I_{t=P_{0} / 4}\right) / I_{t=P_{0} / 4}$ and $-\delta I^{0} / I_{0}=\left(I_{t=P_{0} / 2}-I_{t=P_{0} / 4}\right) / I_{t=P_{0} / 4}$. The shaded areas illustrate the scaled (a) $G_{\lambda_{0}}(T)$ and (b) $\partial G_{\lambda_{0}}(T) / \partial T$ at $n_{\mathrm{e}}=8.5 \cdot 10^{9} \mathrm{~cm}^{-3}$, respectively. The diamond and asterisk denote, respectively, the positive and negative modulations for both Doppler shift and relative intensity.

observed in loops at $T_{\mathrm{i}}=6.4,8.8,12,15$, and $20 \mathrm{MK}$, respectively. The snapshots are taken at $t=P_{0} / 8$ at a viewing angle of $\theta=45^{\circ}$ (other viewing angles give similar results; therefore, they are not shown). At the cooler wing of $\lambda 1118.1$, positive temperature modulation would enhance the intensity, while at the hotter wing, the opposite would occur. The intensity modulation becomes relatively small, as the ambient plasma emission intensity becomes significant or stronger than the loop itself (e.g., $T_{\mathrm{i}}=20 \mathrm{MK}$ ).

Figure $10(\mathrm{~b})$ shows the normalized relative intensity modulation $\left(\delta I^{0} / I_{0}\right) /\left(\delta \rho / \rho_{0}\right)$, which is usually assumed to be 2 (Wang et al. 2009b), at the loop footpoint against loop temperature. It illustrates how the sign of $\partial G_{\lambda_{0}}(T) / \partial T$ would modulate the intensity variation and causes the asymmetry effect (see discussions in Section 4.1.2). We found that $\left(\delta I^{0} / I_{0}\right) /\left(\delta \rho / \rho_{0}\right)$ could be considerably larger than 2 , and that it is only close to 2 at regions where $\left|\partial G_{\lambda_{0}}(T) / \partial T\right|$ is relatively small. At extremely hot loops $(20 \mathrm{MK})$, the normalized intensity modulation could even approach zero.

On the other hand, the Doppler shift velocity of standing slow mode remains detectable for most loop temperatures. However, the normalized amplitude $v_{D}^{0} /\left(v_{z}^{0} \cos \theta\right)$ at the loop apex (it is symmetric for both positive and negative motions) deviates more and more from unity for loops at higher temperatures (Figure 10(a)). This does not mean that the wave energy is undetected. It is still buried in the line width (Figure 9, right column) or the skewness of the spectra, which are not measured in this study.

\section{CONCLUSION}

In this paper, we performed forward modeling to predict the observational features of standing slow modes in hot coronal loops. By considering the geometrical and instrumental effects, we measure the plasma emission intensity, Doppler shift, line width, and spectrum modification caused by the standing slow mode.

We found that the Doppler shift velocity is significantly affected by the LOS effect; it could become undetectable at a viewing angle of $\theta=90^{\circ}$. We found that a linear deprojection by the LOS angle is more accurate than the $\cos \theta$ deprojection as traditionally used in Wang (2011). The emission intensity perturbation is normally a quarter period out of phase with the Doppler shift velocity variation, in both time and space. This effect has been used to identify the standing slow wave mode. Positive temperature variation introduces more emission intensity enhancement than the same amount of negative temperature variation would reduce it, because $\partial G_{\lambda_{0}} /\left.\partial T\right|_{T_{0}^{+}}$is significantly larger than $\partial G_{\lambda_{0}} /\left.\partial T\right|_{T_{0}^{-}}$, i.e., $\partial^{2} G_{\lambda_{0}} /\left.\partial T^{2}\right|_{T_{0}}$ reaches positive extremes. This effect could lead to the halving of the periodicity in intensity and line width at the loop apex. Half periodicity could be also reached if the $\partial G_{\lambda_{0}} /\left.\partial T\right|_{T_{0}^{-}} \gg \partial G_{\lambda_{0}} /\left.\partial T\right|_{T_{0}^{+}}$, which could be only found at the hotter wing of $G_{\lambda_{0}}$, meaning that the loop has to be heated to a few tens of MK in megaflares. This second-order effect in the contribution function could also lead to asymmetry in the emission intensity modulation.

Spectroscopic observations with a sit-and-stare mode alone are not able to resolve longitudinal overtones along the loop, owing to the lack of spatial information. With EUV imagers, the longitudinal overtones could be resolved by investigating the spatial distribution of the emission intensity modulation. A good way of studying standing slow modes is to use joint observations of spectrographs and imagers.

The Doppler shift oscillation of a standing slow wave strongly depends on the viewing angle; for those observations with LOS angles close to $90^{\circ}$, we may not detect Doppler shifts. For loops on the solar disk, a sit-and-stare campaign of spectrographic observation is favored to be placed slightly off the loop apex, because there is a higher probability that the viewing angle would be close to $90^{\circ}$. For spectrographic observations off-limb, most loops' apexes are well exposed for observation at good viewing angles.

The emission contribution function plays a signficant role in determining the observational features of a standing slow wave. It may cause asymmetric intensity modulation for positive and negative temperature perturbations. This effect could be the opposite for loops at the cooler and hotter wings of the $\lambda 1118.1$ line. The normalized relative intensity modulation $\left(\delta I^{0} / I_{0}\right) /\left(\delta \rho / \rho_{0}\right)$ would be 2 only for the loops close to the peak response temperature of the $\lambda 1118.1$ line. The Doppler shift velocity could be significantly smaller than the plasma velocity, if the background plasma emission becomes more significant or stronger than the loop emission. For loops at the hotter wing of a spectral line, the intensity modulation could be small, and one will only observe the Doppler shift. It may lead to a false interpretation of the result, e.g., in the case of a propagating fast wave (Tomczyk et al. 2007; Van Doorsselaere et al. 2008). 
Imaging observations are subject to more variability, e.g., loop curvatures, plasma stratification, and ambiguity of the loop footpoints. The amplitude variation along a loop is sufficient to identify the longitudinal overtones (Figure 8). Normally the $n=1$ mode could be well identified even if the footpoints are hard to find. However, the $n>1$ overtones have shorter wavelength and the nodes close to the footpoints are less reliably measured; therefore, it poses some challenges to identify an $n>1$ overtone.

From the observables, we could attempt to perform MHD seismology (Wang et al. 2007). For the input parameters, we allocate $10 \%$ uncertainties to period $P_{0}$ and loop length $L_{0}$, while we keep the perturbations of density and temperatures as the uncertainties. So we have loop length $L_{0}=100 \pm 10 \mathrm{Mm}$, density $\rho_{0}=(1.4 \pm 0.2) \cdot 10^{-11} \mathrm{~kg} \mathrm{~m}^{-3}$, and temperature $T_{0}=(6.3 \pm 0.5) \mathrm{MK}$, and then we estimate the magnetic field strength $B_{0}=(40.6 \pm 6.2) \mathrm{G}$ inside the loop. If we compare the result to the real input $40 \mathrm{G}$, the main uncertainty is in the estimation of the parameters, while the assumption that $2 L_{0} / P_{0} \simeq C_{\mathrm{T}}$ only results in about $1.5 \%$ of the uncertainty. In the long-wavelength limit, this assumption remains valid and causes small uncertainties in MHD seismology. However, one has to be cautious in using this relation; if the wavelength is much shorter or the plasma $\beta$ is not small, the associated relative error could be estimated by $\left(1+\gamma \beta_{\mathrm{i}} / 2\right)^{0.5}-1$.

The research was supported by an Odysseus grant of the FWO Vlaanderen, the IAP P7/08 CHARM (Belspo), the Topping-Up grant Cor-Seis and the GOA-2015-014 (KU Leuven), and the Open Research Program KLSA201312 of Key Laboratory of Solar Activity of National Astronomical Observatories of China (D.Y.). CHIANTI is a collaborative project involving George Mason University, the University of Michigan (USA), and the University of Cambridge (UK).

\section{REFERENCES}

Andries, J., Arregui, I., \& Goossens, M. 2005, ApJL, 624, L57

Anfinogentov, S., Nakariakov, V. M., Mathioudakis, M., Van Doorsselaere, T., \& Kowalski, A. F. 2013, ApJ, 773, 156

Antolin, P., \& Rouppe van der Voort, L. 2012, ApJ, 745, 152

Antolin, P., \& Van Doorsselaere, T. 2013, A\&A, 555, A74

Antolin, P., \& Verwichte, E. 2011, ApJ, 736, 121

Antolin, P., Vissers, G., Pereira, T. M. D., Rouppe van der Voort, L., \& Scullion, E. 2015, ApJ, 806, 81

Antolin, P., Yokoyama, T., \& van Doorsselaere, T. 2014, ApJL, 787, L22

Arregui, I. 2015, RSPTA, 373, 2042

Arregui, I., Andries, J., van Doorsselaere, T., Goossens, M., \& Poedts, S. 2007, A\&A, 463, 333

Asai, A., Shimojo, M., Isobe, H., et al. 2001, ApJL, 562, L103

Aschwanden, M. J. 2005, Physics of the Solar Corona: An Introduction with Problems and Solutions (2nd ed.; Berlin: Springer-Verlag)

Aschwanden, M. J., Fletcher, L., Schrijver, C. J., \& Alexander, D. 1999, ApJ, 520,880

Aschwanden, M. J., Nightingale, R. W., Andries, J., Goossens, M., \& Van Doorsselaere, T. 2003, ApJ, 598, 1375

Boerner, P., Edwards, C., Lemen, J., et al. 2012, SoPh, 275, 41

Chen, Y., Feng, S. W., Li, B., et al. 2011, ApJ, 728, 147

Chen, Y., Song, H. Q., Li, B., et al. 2010, ApJ, 714, 644

Cooper, F. C., Nakariakov, V. M., \& Tsiklauri, D. 2003a, A\&A, 397, 765

Cooper, F. C., Nakariakov, V. M., \& Williams, D. R. 2003b, A\&A, 409, 325

De Moortel, I., Hood, A. W., Ireland, J., \& Walsh, R. W. 2002a, SoPh, 209, 89

De Moortel, I., Ireland, J., \& Walsh, R. W. 2000, A\&A, 355, L23

De Moortel, I., Ireland, J., Walsh, R. W., \& Hood, A. W. 2002b, SoPh, 209, 61

De Moortel, I., \& Nakariakov, V. M. 2012, RSPTA, 370, 3193

Del Zanna, G., O'Dwyer, B., \& Mason, H. E. 2011, A\&A, 535, A46

Dere, K. P., Landi, E., Mason, H. E., Monsignori Fossi, B. C., \& Young, P. R. 1997, A\&AS, 125, 149
Edwin, P. M., \& Roberts, B. 1983, SoPh, 88, 179

Fleishman, G. D., \& Kuznetsov, A. A. 2010, ApJ, 721, 1127

Goossens, M., Erdélyi, R., \& Ruderman, M. S. 2011, SSRv, 158, 289

Gruszecki, M., Nakariakov, V. M., \& Van Doorsselaere, T. 2012, A\&A, 543, A12

Hood, A. W., Browning, P. K., \& van der Linden, R. A. M. 2009, A\&A, 506, 913

Jess, D. B., Reznikova, V. E., van Doorsselaere, T., Keys, P. H., \& Mackay, D. H. 2013, ApJ, 779, 168

Kim, S., Nakariakov, V. M., \& Shibasaki, K. 2012, ApJL, 756, L36

Klimchuk, J. A. 2006, SoPh, 234, 41

Krishna Prasad, S., Banerjee, D., \& Van Doorsselaere, T. 2014, ApJ, 789, 118

Kumar, P., Innes, D. E., \& Inhester, B. 2013, ApJL, 779, L7

Kumar, P., Nakariakov, V. M., \& Cho, K.-S. 2015, ApJ, 804, 4

Kupriyanova, E. G., Melnikov, V. F., \& Shibasaki, K. 2013, SoPh, 284, 559

Kuznetsov, A. A., van Doorsselaere, T., \& Reznikova, V. E. 2015, SoPh, 290,1173

Landi, E., Young, P. R., Dere, K. P., Del Zanna, G., \& Mason, H. E. 2013, ApJ, 763, 86

Mariska, J. T. 2006, ApJ, 639, 484

Melnikov, V. F., Reznikova, V. E., Shibasaki, K., \& Nakariakov, V. M. 2005, A\&A, 439, 727

Moreels, M. G., \& Van Doorsselaere, T. 2013, A\&A, 551, A137

Mossessian, G., \& Fleishman, G. D. 2012, ApJ, 748, 140

Nakariakov, V. M., \& Melnikov, V. F. 2009, SSRv, 149, 119

Nakariakov, V. M., \& Ofman, L. 2001, A\&A, 372, L53

Nakariakov, V. M., Ofman, L., Deluca, E. E., Roberts, B., \& Davila, J. M. 1999, Sci, 285, 862

Nakariakov, V. M., \& Verwichte, E. 2005, LRSP, 2, 3

Ning, Z. 2014, SoPh, 289, 1239

Ofman, L. 2010, LRSP, 7, 4

Ofman, L., \& Wang, T. 2002, ApJL, 580, L85

Parnell, C. E., \& De Moortel, I. 2012, RSPTA, 370, 3217

Peter, H., Bingert, S., Klimchuk, J. A., et al. 2013, A\&A, 556, A104

Poduval, B., DeForest, C. E., Schmelz, J. T., \& Pathak, S. 2013, ApJ, 765, 144

Priest, E. R., Heyvaerts, J. F., \& Title, A. M. 2002, ApJ, 576, 533

Reale, F. 2014, LRSP, 11, 4

Reznikova, V. E., Antolin, P., \& van Doorsselaere, T. 2014, ApJ, 785, 86

Reznikova, V. E., van Doorsselaere, T., \& Kuznetsov, A. A. 2015, A\&A, 575, A47

Ruderman, M. S. 2011a, A\&A, 534, A78

Ruderman, M. S. 2011b, SoPh, 271, 41

Ruderman, M. S., \& Erdélyi, R. 2009, SSRv, 149, 199

Sakurai, T., Goossens, M., \& Hollweg, J. V. 1991, SoPh, 133, 227

Sakurai, T., Ichimoto, K., Raju, K. P., \& Singh, J. 2002, SoPh, 209, 265

Scullion, E., Rouppe van der Voort, L., Wedemeyer, S., \& Antolin, P. 2014, ApJ, 797, 36

Selwa, M., Murawski, K., \& Solanki, S. K. 2005, A\&A, 436, 701

Taroyan, Y., \& Bradshaw, S. 2008, A\&A, 481, 247

Taroyan, Y., \& Erdélyi, R. 2009, SSRv, 149, 229

Taroyan, Y., Erdélyi, R., Doyle, J. G., \& Bradshaw, S. J. 2005, A\&A, 438, 713

Taroyan, Y., Erdélyi, R., Wang, T. J., \& Bradshaw, S. J. 2007, ApJL, 659, L173

Tomczyk, S., McIntosh, S. W., Keil, S. L., et al. 2007, Sci, 317, 1192

van der Holst, B., Sokolov, I. V., Meng, X., et al. 2014, ApJ, 782, 81

Van Doorsselaere, T., de Groof, A., Zender, J., Berghmans, D., \& Goossens, M. 2011a, ApJ, 740, 90

Van Doorsselaere, T., Gijsen, S. E., Andries, J., \& Verth, G. 2014, ApJ, 795, 18

Van Doorsselaere, T., Nakariakov, V. M., \& Verwichte, E. 2008, ApJL, 676, L73

Van Doorsselaere, T., Wardle, N., del Zanna, G., et al. 2011b, ApJL, 727, L32

Verth, G., Erdélyi, R., \& Jess, D. B. 2008, ApJL, 687, L45

Wang, T. 2011, SSRv, 158, 397

Wang, T., Innes, D. E., \& Qiu, J. 2007, ApJ, 656, 598

Wang, T., Solanki, S. K., Curdt, W., Innes, D. E., \& Dammasch, I. E. 2002, ApJL, 574, L101

Wang, T. J., Ofman, L., \& Davila, J. M. 2009a, ApJ, 696, 1448

Wang, T. J., Ofman, L., Davila, J. M., \& Mariska, J. T. 2009b, A\&A, 503, L25

Wang, T. J., Solanki, S. K., Curdt, W., et al. 2003a, A\&A, 406, 1105

Wang, T. J., Solanki, S. K., Innes, D. E., \& Curdt, W. 2005, A\&A, 435, 753

Wang, T. J., Solanki, S. K., Innes, D. E., Curdt, W., \& Marsch, E. 2003b, A\&A, 402, L17

Williams, D. R., Mathioudakis, M., Gallagher, P. T., et al. 2002, MNRAS, 336, 747

Williams, D. R., Phillips, K. J. H., Rudawy, P., et al. 2001, MNRAS, 326, 428 
Winebarger, A. R., Warren, H., van Ballegooijen, A., DeLuca, E. E., \& Golub, L. 2002, ApJL, 567, L89

Yuan, D., \& Nakariakov, V. M. 2012, A\&A, 543, A9

Yuan, D., Nakariakov, V. M., Huang, Z., et al. 2014a, ApJ, 792, 41
Yuan, D., Pascoe, D. J., Nakariakov, V. M., Li, B., \& Keppens, R. 2015, ApJ, 799, 221

Yuan, D., Sych, R., Reznikova, V. E., \& Nakariakov, V. M. 2014b, A\&A, 561, A19 